\title{
Chronic radiation exposure of neuroblastoma cells reduces nMYC copy number
}

\author{
MANU GNANAMONY ${ }^{1}$, REUBEN ANTONY ${ }^{2}$, KAREN S. FERNÁNDEZ $^{3}$, LIBES JAIME ${ }^{1}$, \\ JULIAN LIN ${ }^{4}$, PUSHPA A. JOSEPH ${ }^{5}$ and CHRISTOPHER S. GONDI ${ }^{5,6,7}$ \\ ${ }^{1}$ Department of Pediatrics, University of Illinois College of Medicine, Peoria, IL 61605; \\ ${ }^{2}$ UC Davis Comprehensive Cancer Center, Pediatric Hematology and Oncology, UC Davis Children's Hospital, \\ Sacramento, CA 95817; ${ }^{3}$ Cancer and Blood Diseases Center, 9300 Valley Children's Place FC13, Madera, CA 93636; \\ Departments of ${ }^{4}$ Neurosurgery, ${ }^{5}$ Pathology, ${ }^{6}$ Internal Medicine and ${ }^{7}$ Surgery, \\ University of Illinois College of Medicine, Peoria, IL 61605, USA
}

Received October 25, 2016; Accepted March 3, 2017

DOI: $10.3892 / 01.2017 .6652$

\begin{abstract}
Neuroblastoma accounts for $>15 \%$ of cancer-associated mortalities of children in the USA. Despite aggressive treatment regimens, the long-term survival for these children remains $<40 \%$. The identification of $\mathrm{v}-\mathrm{Myc}$ avian myelocytomatosis viral oncogene neuroblastoma-derived homolog (nMYC) gene amplification during diagnosis is associated with poor prognosis in neuroblastoma. There are limited studies examining changes in nMYC copy numbers in response to therapy and its biological effect on cancer cells. The aim of the present study was to evaluate the effect of radiation on nMYC expression and amplification status in high-risk neuroblastoma. The effect of acute (5 Gy) and chronic (25 Gy) radiation on two nMYC-amplified cell lines, SK-N-BE (2) and NB-1691, was investigated. The results demonstrate that, following chronic but not acute radiation, the two cell lines regained their proliferation potential similar to the controls. This increased proliferation was characterized by loss of nMYC mRNA and protein expression. It was also revealed that nMYC loss was accompanied by nuclear localization of c-Myc. Using fluorescent in situ hybridization and quantitative polymerase chain reaction analysis, the results of the present study demonstrated that chronic radiation causes a severe loss of nMYC gene copy number. The present study is the first to provide experimental evidence that prolonged radiation therapy affects nMYC gene copy number in high-risk neuroblastoma but does not significantly improve the prognostic outlook.
\end{abstract}

Correspondence to: Dr Christopher S. Gondi, Department of Internal Medicine, University of Illinois College of Medicine at Peoria, 1 Illini Drive, Peoria, IL 61605, USA

E-mail: gondi@uic.edu

Key words: neuroblastoma, v-Myc avian myelocytomatosis viral oncogene neuroblastoma-derived homolog, copy number, radiation

\section{Introduction}

v-Myc avian myelocytomatosis viral oncogene neuroblastoma-derived homolog (MYCN), also known as nMYC, was first described in 1983 as a gene homologous with vMYC, but is distinct from the classical v-myc avian myelocytomatosis viral oncogene homolog (cMYC) proto-oncogene and is highly amplified in high-risk neuroblastoma (1). Although nMYC and cMYC genes share high levels of homology in their coding regions and their protein products, they vary widely in their tissue expression profiles (2). According to the International Neuroblastoma Risk Group, the most established risk factors for high-risk neuroblastoma are age, histology, grade of tumor differentiation, chromosome 11q status, DNA ploidy and $\mathrm{nMYC}$ amplification status (3). nMYC amplification is observed in $\sim 25 \%$ of neuroblastoma cases and is associated with poor prognosis and is also considered the best-known genetic predictor for neuroblastoma risk (4). Additionally, nMYC amplification status is also used as a diagnostic tool to determine the prognostic outcomes of neuroblastoma treatments (5).

Radiation therapy is one of the standard therapies used for the treatment of recurrent high-risk neuroblastoma. Radiation therapy has led to significant success and a previous study has illustrated that patients treated using 21 to $24 \mathrm{~Gy}$ radiation at the local tumor sites resulted in $94 \%$ local control, $66 \%$ event-free survival and $86 \%$ overall survival, making it a vital treatment strategy for the treatment of neuroblastoma (6). However, a significant proportion of recipients continue to exhibit recurrence. Radiation affects cells primarily by inducing DNA damage and cells have evolved repair mechanisms to counter this damage. Numerous mechanisms of radiation resistance have evolved, which include activation of heat-shock proteins and an alteration of response signaling pathways that promote cell survival (7).

In order to determine whether neuroblastoma cells that are capable of surviving high doses of radiation exhibit changes in expression of nMYC, the effect of low-dose (5 Gy) and high-dose $(25 \mathrm{~Gy})$ radiation on neuroblastoma cells was 
studied. The results demonstrate that chronic but not acute radiation results in the loss of $\mathrm{nMYC}$ mRNA and protein expression and loss of nMYC gene copy number. A further unidentified mechanism by which neuroblastoma cells may adapt to radiation by actively suppressing genomic copy number was also demonstrated.

\section{Materials and methods}

Cell lines and culture conditions. The neuroblastoma cell line SK-N-BE (2) was obtained from the American Type Culture Collection (Manassas, VA, USA) and the NB1691 cell line was provided by Dr Peter Houghton (St. Jude Children's Research Hospital, Memphis, TN, USA). Cells were cultured in RPMI medium with $10 \%$ fetal bovine serum and $1 \%$ penicillin/streptomycin at $37^{\circ} \mathrm{C}$ in a humidified atmosphere containing $5 \% \mathrm{CO}_{2}$. The two cell lines were screened to be free of Mycoplasma contamination using a polymerase chain reaction (PCR) Mycoplasma detection kit (ABM, Inc., Richmond, BC, Canada). Human umbilical vein endothelial cells (HUVECs) were obtained from Thermo Fisher Scientific, Inc. (Waltham, MA, USA) and cultured in Medium 200 supplemented with $1 \mathrm{X}$ low-serum growth medium. Low passage number (3) HUVECs were used for the tube formation assay.

Antibodies. Antibodies were obtained from the following sources: cMYC (cat. no. 13987s), nMYC (cat. no. 9405s) and anti-mouse IgG (cat. no. 7076s) from Cell Signaling Technologies, Inc. (Danvers, MA, USA), $\beta$-actin (cat. no. sc47778) and anti-rabbit IgG (cat. no. sc2030) from Santa Cruz Biotechnology, Inc. (Dallas, TX, USA).

Radiation treatment. Radiation was performed using the RS2000 radiator (Rad Source Technologies, Inc., Boca Raton, FL, USA). For acute radiation, a single dose of 5 Gy was administered and cells were processed for all the experiments. Chronic radiation treatment is discussed as follows.

Generation of radiation-resistant cells. SK-N-BE (2) and NB-1691 cells were treated with 5 Gy radiation and returned to the $\mathrm{CO}_{2}$ incubator and grown at $37^{\circ} \mathrm{C}$ in a humidified atmosphere. Radiosensitive cells died following between 3 and 5 days and the surviving cells grew as colonies. These colonies were then carefully expanded until they became confluent. These cells were then radiated again with 5 Gy and the cycle was continued until the cells were treated with a cumulative dosage of $25 \mathrm{~Gy}$. The surviving cells were designated as chronic radiated cells and used for additional experiments. This was performed to recapitulate the clinical radiation dosage regime. Radiation-resistant cells were termed chronic radiation cells.

Colony formation assay. Cells were counted, and 1,000 cells were plated in $60-\mathrm{mm}$ plates. The plates were incubated at $37^{\circ} \mathrm{C}$ for 2 weeks with the medium changed every 3 days. The cells were then fixed with acetic acid/methanol and stained with $0.5 \%$ crystal violet for $2 \mathrm{~h}$. The plates were de-stained with water, air-dried and imaged. The assay was performed in triplicate.
Cell viability assay. A total of 1,000 cells were plated in 96-well plates and incubated for $24,48,72$ and $96 \mathrm{~h}$ at $37^{\circ} \mathrm{C}$. MTT was added to a final concentration of $0.5 \mathrm{mg} / \mathrm{ml}$ and incubated for an additional $2 \mathrm{~h}$ at $37^{\circ} \mathrm{C}$. The reaction was stopped by adding $100 \mu \mathrm{l}$ dimethyl sulfoxide for $30 \mathrm{~min}$ and the absorbance was read at $550 \mathrm{~nm}$ using a spectrophotometer. Untreated wild-type cells served as the control group. A total of 12 replicates were included for each time point and the mean percentage proliferation was plotted.

Reverse transcription-quantitative PCR (RT-qPCR). Total RNA was isolated from cells using TRIzol (Thermo Fisher Scientific, Inc.) and converted into cDNA using an iScript cDNA synthesis kit (Bio-Rad Laboratories, Inc., Hercules, CA, USA), according to the manufacturer's protocol. Amplification of cDNA was performed using iTaq Universal SYBR-Green Supermix (Bio-Rad Laboratories, Inc.) using primers targeting $\mathrm{cMYC}$ and $\mathrm{nMYC}$, and normalized against hypoxanthine-guanine phosphoribosyltransferase (HPRT). The primer sequences used were as follows: nMYC forward, 5'-CACAAGGCCCTCAGTACCTC-3' and reverse, 5'-ACC ACGTCGATTTCTTCCTC-3'; cMYC forward, 5'-CGTCTC CACACATCAGCACAA-3' and reverse, 5'-CACTGTCCA ACTTGACCCTCTTG-3'; HPRT forward, 5'-TGACACTGG CAAAACAATGCA-3' and reverse 5'-GGTCCTTTTCAC CAGCAAGCT-3'. The thermocycling conditions were as follows: Initial denaturation at $95^{\circ} \mathrm{C}$ for $30 \mathrm{sec}$, followed by 40 cycles of $95^{\circ} \mathrm{C}$ for $5 \mathrm{sec}$ and $60^{\circ} \mathrm{C}$ for $30 \mathrm{sec}$. A total of 4 replicates/sample were performed. mRNA levels were quantified using the $2^{-\Delta \Delta \mathrm{Cq}}$ method and plotted as arbitrary units according to standard protocols on Microsoft Excel (Microsoft Corporation, Redmond, WA, USA) (8).

Western blotting. Whole cell lysates were prepared by treating cells with M-PER mammalian protein extraction reagent (Thermo Fisher Scientific, Inc.) and protein concentration was measured using Pierce $660 \mathrm{~nm}$ protein assay reagent (Thermo Fisher Scientific, Inc.). Equal concentrations of protein $(40 \mu \mathrm{g})$ were separated by SDS-PAGE (10\% gels) and transferred onto polyvinylidene fluoride membranes. Blots were blocked with 5\% skimmed milk in PBS containing Tween-20 (PBST) for $1 \mathrm{~h}$ at room temperature, probed with appropriate primary antibody (1:1,000 dilution) in PBST overnight at $4^{\circ} \mathrm{C}$ and secondary antibodies (1:5,000 dilution) for $1 \mathrm{~h}$ at room temperature. Protein bands were visualized using Pierce enhanced chemiluminescence western blot substrate (Thermo Fisher Scientific, Inc.), and $\beta$-actin was used as a loading control. Western blot analysis was performed to confirm RT-PCR results.

Confocal microscopy. Control, acute and chronic radiation-treated cells were plated in 8 -well chamber slides. After $24 \mathrm{~h}$, cells were fixed in formaldehyde for $10 \mathrm{~min}$ and permeabilized with $0.1 \%$ Igepal-300. The slides were blocked with $1 \%$ BSA in PBST for 30 min and probed with primary antibody (cMYC or nMYC) for $1 \mathrm{~h}$ at room temperature. The slides were washed and treated with appropriate fluorescent secondary antibody for $30 \mathrm{~min}$. Nuclear staining was performed using propidium iodide (BioSure ${ }^{\circledR}$, Grass Valley, CA, USA) and slides were mounted using Vectashield mounting medium (Vector Laboratories, Inc., Burlingame, 
CA, USA). Images were captured using a confocal microscope (Olympus Corporation, Center Valley, PA, USA).

nMYC gene amplification/copy number analysis. Copy number analysis was performed using the comparative $\mathrm{Cq}$ method as previously described (9). Genomic DNA was isolated from the control and the treated cells using the DNA mini kit (Qiagen, Inc., Valencia, CA, USA) and amplification was performed using iTaq Universal SYBR-Green Supermix (Bio-Rad Laboratories, Inc.). The thermal cycling conditions maintained were as follows: Initial denaturation at $95^{\circ} \mathrm{C}$ for $30 \mathrm{sec}$, followed by 40 cycles of $95^{\circ} \mathrm{C}$ for $5 \mathrm{sec}$ and $60^{\circ} \mathrm{C}$ for $30 \mathrm{sec}$. A total of four replicates were included for each sample, and the copy number was calculated by normalizing against a calibrator DNA sample that possessed a disomic copy number of genes (control genomic DNA, Applied Biosystems; Thermo Fisher Scientific, Inc.). The mean copy number of two reference genes, B-cell maturation antigen (BCMA) and syndecan 4 (SDC4), was used for normalization. The copy number of nMYC was calculated using the formula:.

$$
2^{-\Delta \Delta \mathrm{Cq}}=\frac{(1+E)^{-\Delta \mathrm{Cq} \text { gene }}}{(1+E)^{-\Delta \mathrm{Cq} \text { reference }}}
$$

where E represents efficiency, set at $0.95 ; \Delta \mathrm{Cq}$ gene represents the difference in $\mathrm{Cq}$ value between test sample and calibrator sample for the target gene; $\Delta \mathrm{Cq}$ reference represents the difference in $\mathrm{Cq}$ value between test sample and calibrator sample for the reference gene.

The primer sequences used were as follows: nMYC forward, 5'-CGCAAA AGC CAC CTC TCA T TA-3'; reverse, 5'-TCCAGCAGATGCCACATAAGG-3'; BCMA forward, 5'-CGACTCTGACCATTGCTT TCC-3' and reverse, 5'-AAGCAGCTGGCAGGCTCTT-3' and SDC4 forward, 5'-CAGGGTCTGGGAGCCAAGT-3' and reverse, 5'-GCACAGTGCTGGACATTGACA-3'.

Fluorescent in-situ hybridization (FISH). FISH was performed in cells using the nMYC amplification probe (red) spanning the nMYC gene in chromosome 2 position $2 \mathrm{p} 24.3$ (Cytocell, Cambridge, UK). Control and treated cells were plated in 8-well chamber slides. After $24 \mathrm{~h}$, the cells were fixed using methanol: acetic acid for $5 \mathrm{~min}$, denatured by heating at $75^{\circ} \mathrm{C}$ for $5 \mathrm{~min}$ and hybridized with probe at $37^{\circ} \mathrm{C}$ overnight according to the manufacturer's protocol. The slides were washed, mounted and visualized using a confocal microscope. A control probe (green) targeting lymphoid nuclear protein related to AF4 gene at chromosome 2 position 2 q11.2 was included in the probe mix and served as an internal control for chromosome number.

Conditioned medium collection. NB-1691 and SK-N-BE (2) wild-type, acute and chronic radiation treated cells were plated in $60 \mathrm{~mm}$ cell culture plates and grown in RPMI 1640 medium (cat. no. MT10040CV; Thermo Fisher Scientific Inc.) with $10 \%$ FBS and $1 \%$ penicillin streptomycin. When the cells reached $\sim 80 \%$ confluence, they were washed with RPMI 1640 medium without FBS and antibiotics, resuspended in $2 \mathrm{ml}$ serum-free medium and incubated overnight in a humidified $37^{\circ} \mathrm{C}$ incubator. The conditioned medium was collected and debris removed by centrifugation at $800 \mathrm{x}$ g for $5 \mathrm{~min}$ at room temperature. The supernatant was separated and used for subsequent angiogenesis assays.

In vitro angiogenesis assay. The angiogenesis assay was performed using the HUVEC tube formation assay. HUVECs were first stained with a cell-permeant fluorescent dye, calcein acetoxymethyl ester (AM) (Thermo Fisher Scientific, Inc.) at a concentration of $2 \mu \mathrm{g} / \mathrm{ml}$ and incubated at $37^{\circ} \mathrm{C}$ in the dark for at least $30 \mathrm{~min}$. A total of $40 \mu \mathrm{l}$ of Geltrex basement membrane matrix (Thermo Fisher Scientific, Inc.) was coated on 96-well plates and allowed to solidify for $30 \mathrm{~min}$. HUVECs were trypsinized and resuspended in SFM. Cells were plated at a concentration of 20,000 cells/well in $100 \mu \mathrm{l}$ appropriate conditioned medium. Cells with complete medium were used as a positive control and cells with SFM were used as negative control. The plate was incubated at $37^{\circ} \mathrm{C}$ in a humidified incubator for $3 \mathrm{~h}$. Tube formation was visualized using an inverted fluorescence microscope at x10 magnification.

In vitro invasion assay. Invasion was performed using the Oris Pro 96-well invasion assay kit (Platypus Technologies, Madison, WI, USA) as described below. A total of 20,000 calcein AM-treated cells were plated in collagen I-coated 96-well plates and incubated for $1 \mathrm{~h}$ in a humidified $37^{\circ} \mathrm{C}$ incubator. The cells were overlaid with collagen I and incubated for an additional $1 \mathrm{~h}$ in a humidified $37^{\circ} \mathrm{C}$ incubator. Following polymerization, $100 \mu \mathrm{l}$ complete medium was added to all the wells. Pre-invasion images were captured using an inverted fluorescent microscope at $\mathrm{x} 10$ magnification. The plate was then incubated at $37^{\circ} \mathrm{C}$ for $48 \mathrm{~h}$. After $48 \mathrm{~h}$, post-invasion images were also captured.

Statistical analysis. All statistical analysis was performed using GraphPad Prism (version 6.0; GraphPad Software, Inc., La Jolla, CA, USA). Student's t-tests were used to compare significance between study groups. $\mathrm{P}<0.05$ was considered to indicate a statistically significant difference.

\section{Results}

Chronic radiation increases cell viability and survival. To test the effect of radiation on neuroblastoma cells, NB1691 and SK-N-BE (2) cells were exposed to acute radiation (single 5 Gy dose) and chronic radiation (5 doses of 5 Gy each, cumulative dosage of $25 \mathrm{~Gy}$ ). It was revealed that cells resistant to chronic radiation were able to form colonies, whereas control cells that were exposed to acute radiation ( $5 \mathrm{~Gy}$ ) exhibited little to no anchorage-dependent proliferation (Fig. 1A). Additionally, to determine whether cells exposed to acute radiation and resistant cells exposed to chronic radiation exhibited any change in proliferative potential, an MTT assay was performed. It was observed that over a period of $96 \mathrm{~h}$, the proliferative potential of radiation-resistant cells was similar, if not greater, compared with un-radiated controls, whereas cells exposed to acute radiation demonstrated little to no proliferation (Fig. 1B).

Chronic radiation induces loss of $n M Y C$ expression. Fig. $1 \mathrm{~B}$ indicates that neuroblastoma cells exposed to chronic radiation exhibited high proliferation rates when compared with cells 
A
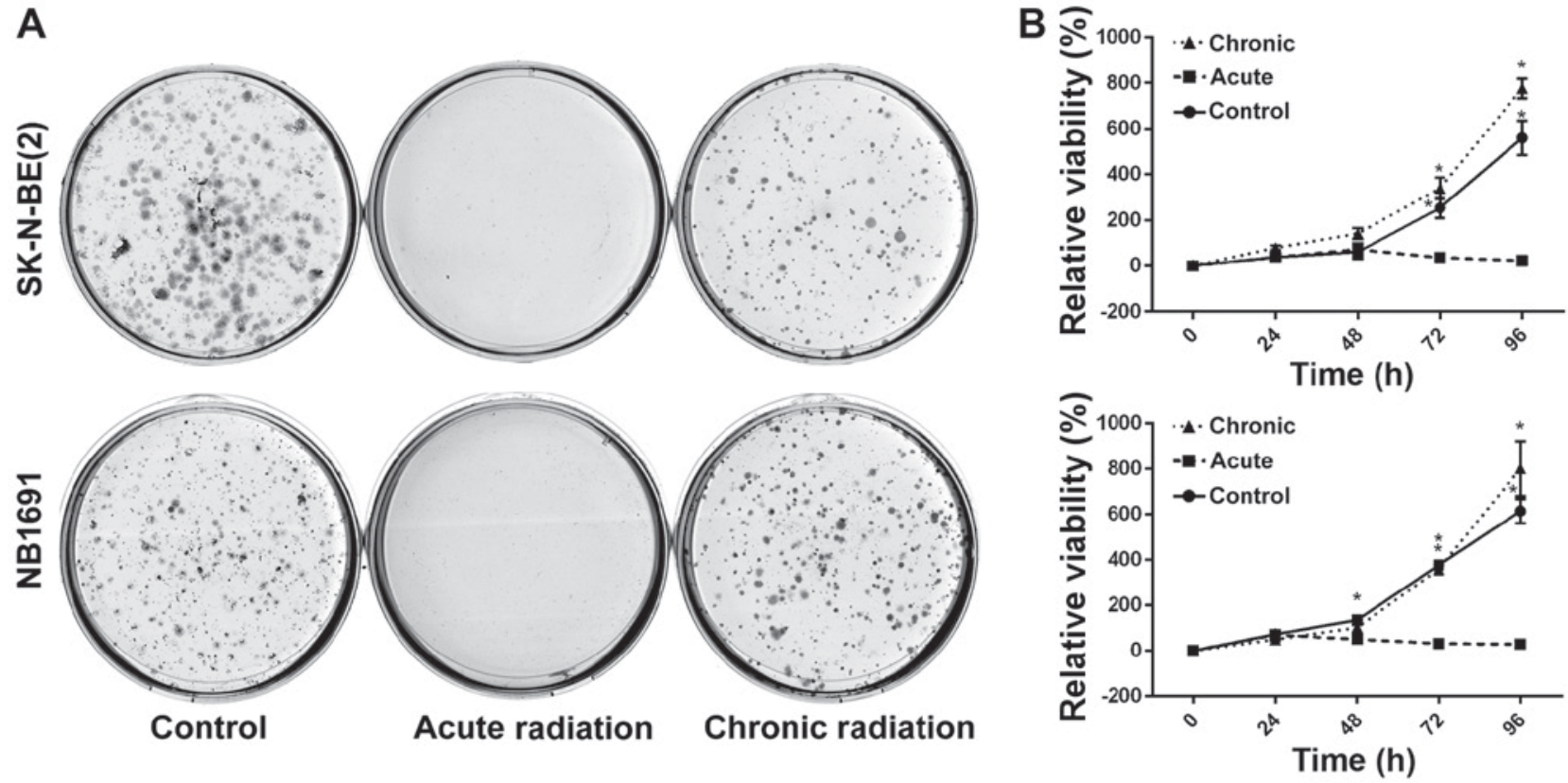

Figure 1. Effect of chronic radiation on cell proliferation and survival of neuroblastoma cells. (A) The ability of neuroblastoma cells to survive and form colonies were measured using a colony formation assay. Acute radiation resulted in reduced colonies compared with controls, whereas following chronic radiation, the cells regained the ability to form colonies. (B) Cell viability was measured at 24, 48, 72 and $96 \mathrm{~h}$ using an MTT assay. NB1691 and SK-N-BE (2) cells treated with chronic radiation demonstrated increased proliferation potential compared with acute radiation and controls $\left({ }^{*} \mathrm{P}<0.0001 ;{ }^{* *} \mathrm{P}=0.004\right)$.

A

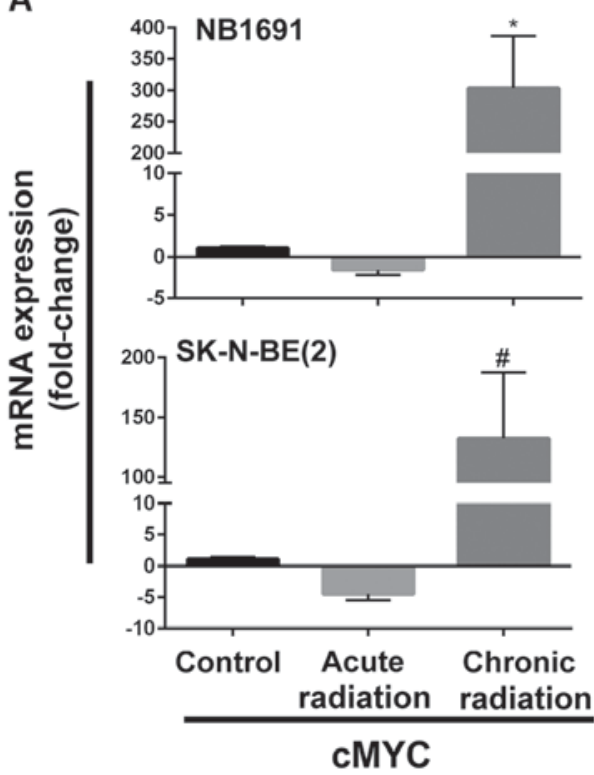

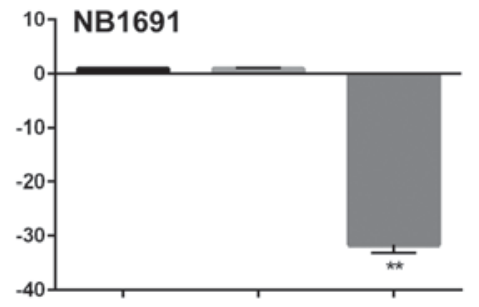

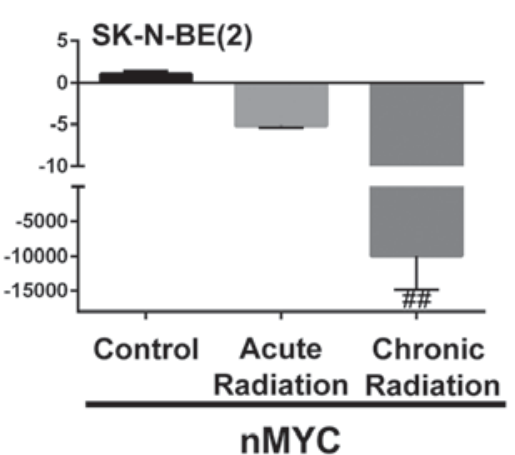

B
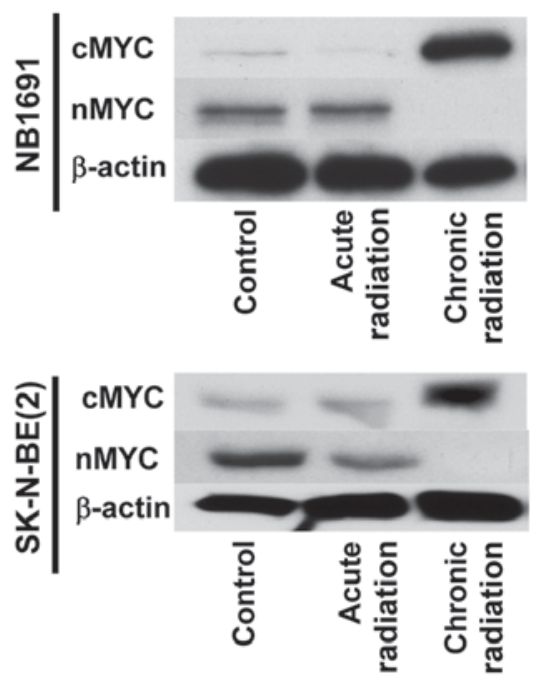

Figure 2. Effect of chronic radiation on nMYC and cMYC expression. (A) Reverse transcription-quantitative polymerase chain reaction analysis identified decreased expression of nMYC mRNA and increased expression of cMYC mRNA in NB1691 and SK-N-BE (2) cells treated with chronic radiation ("P=0.0003; ${ }^{* *} \mathrm{P}<0.0001 ;{ }^{\#} \mathrm{P}=0.004 ;{ }^{\# \#} \mathrm{P}=0.009$ ). (B) Western blot analysis demonstrating a decrease in nMYC protein levels and an increase in cMYC protein levels in cells treated with chronic radiation. nMYC, v-Myc avian myelocytomatosis viral oncogene neuroblastoma-derived homolog; cMYC, v-Myc avian myelocytomatosis viral oncogene neuroblastoma.

exposed to acute radiation. It is known that increased expression of nMYC is associated with increased proliferation (10). Therefore, in an attempt to determine whether chronic radiation influenced the expression levels of nMYC expression, RT-qPCR analysis was used to determine the expression levels of nMYC and cMYC. It was observed that radiation-resistant (chronic) cells exhibited a significant loss of nMYC mRNA expression in the NB1691 and SK-N-BE (2) cell lines. Additionally, expression levels of cMYC mRNA were increased in the two NB1691 and SK-N-BE (2) cells exposed to chronic radiation (Fig. 2A). This change was additionally corroborated and validated with mRNA expression levels with protein expression levels of nMYC and cMYC. It was observed that a decrease in nMYC mRNA corresponded to a decrease in nMYC protein levels 

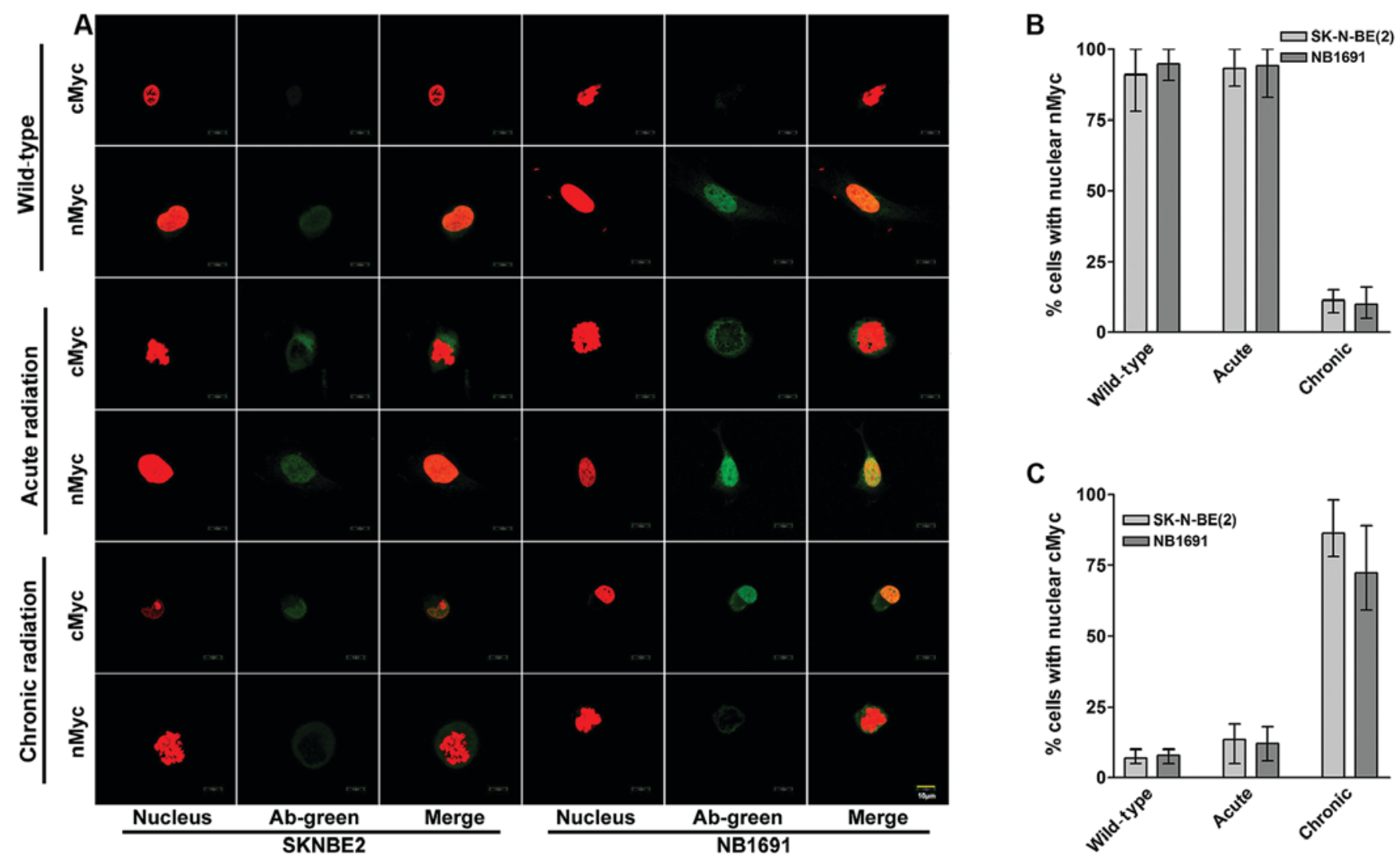

Figure 3. Nuclear localization of cMYC in neuroblastoma cells treated with chronic radiation. (A) Localization of nMYC and cMYC proteins during radiation was identified using confocal fluorescent microscopy at X10 magnification. Representative images illustrating nMYC and cMYC counterstained with propidium iodide. Under normal and acute radiation conditions, nMYC is observed to be localized in the nucleus, whereas cMYC is either absent or observed in low concentrations in the cytoplasm. Chronic radiation resulted in a significant increase in localization of cMYC in the nucleus, which is associated with a decrease in $\mathrm{nMYC}$ expression (scale bar, $10 \mu \mathrm{m}$ ). Cells were counted in five random fields and the average percentage of cells exhibiting nMYC and cMYC expression were determined. (B) There was a significant decrease in cells expressing nMYC in the nucleus and (C) an increase in cells expressing nuclear cMYC when treated with chronic radiation. Red staining indicates the nucleus; green staining indicates nMYC and cMYC. nMYC, v-Myc avian myelocytomatosis viral oncogene neuroblastoma derived homolog; cMYC, v-Myc avian myelocytomatosis viral oncogene homolog; Ab, antibody.

and an increase in cMYC mRNA levels corresponded to an increase in cMYC protein levels in NB1691 and SK-N-BE (2) cells exposed to chronic radiation (Fig. 2B).

cMYC localizes in the nucleus of chronic radiation cells. To study the cellular localization of $\mathrm{cMYC}$ and $\mathrm{nMYC}$ proteins in acute and chronic radiation cells, confocal microscopy was performed for cMYC and nMYC in the NB1691 and SK-N-BE (2) cell lines. It was observed that in controls and acute radiation cells, high expression of nMYC was predominantly exhibited in the nucleus, whereas lower expression of cMYC was exhibited in the cytoplasm. Notably, chronic radiation cells demonstrated a significant loss of nMYC expression in the nucleus, which was compensated by a corresponding increase in the expression of cMYC (Fig. 3A). Quantitative analysis demonstrated that only $11 \%$ of SK-N-BE (2) cells and $10 \%$ of NB-1691 cells expressed nMYC in the nucleus of chronic radiation cells compared with $80 \%$ in control SK-N-BE (2) and 84\% in NB-1691 control cells (Fig. 3B). In contrast, following treatment with chronic radiation, $\mathrm{cMYC}$ expression was observed in the nucleus of $86 \%$ SK-N-BE (2) cells and $72 \%$ NB-1691 cells compared with 7 and $8 \%$ in their respective controls (Fig. 3C).
Chronic radiation induces loss of MYCN gene amplification. Amplification of the nMYC gene in neuroblastoma has been identified to be markedly associated with advanced disease stage (5). A mechanism by which radiation kills tumor cells is by DNA damage. In the present study, initial experiments demonstrated a loss of nMYC mRNA and protein expression in cells that were exposed to chronic radiation, the effect of radiation on nMYC gene amplification using FISH and qPCR based gene copy number analysis was examined. From the FISH analysis, it was observed that the nMYC gene copy number was significantly decreased in chronic radiation cells when compared with controls or acute radiation cells (Fig. 4A). The FISH results were confirmed by a qPCR-based copy number analysis that also demonstrated that chronic radiation resulted in significant loss of nMYC copy number in NB1691 and SK-N-BE (2) cells (Fig. 4B).

Chronic radiation inhibits angiogenesis and invasion in neuroblastoma cells. Using the well-established HUVEC tube formation assay, the effect of radiation on neuroblastoma cells was investigated. The results revealed that chronic, but not acute, radiation resulted in significant inhibition of secretion of pro-angiogenic factors (Fig. 5A). The results also demonstrated 

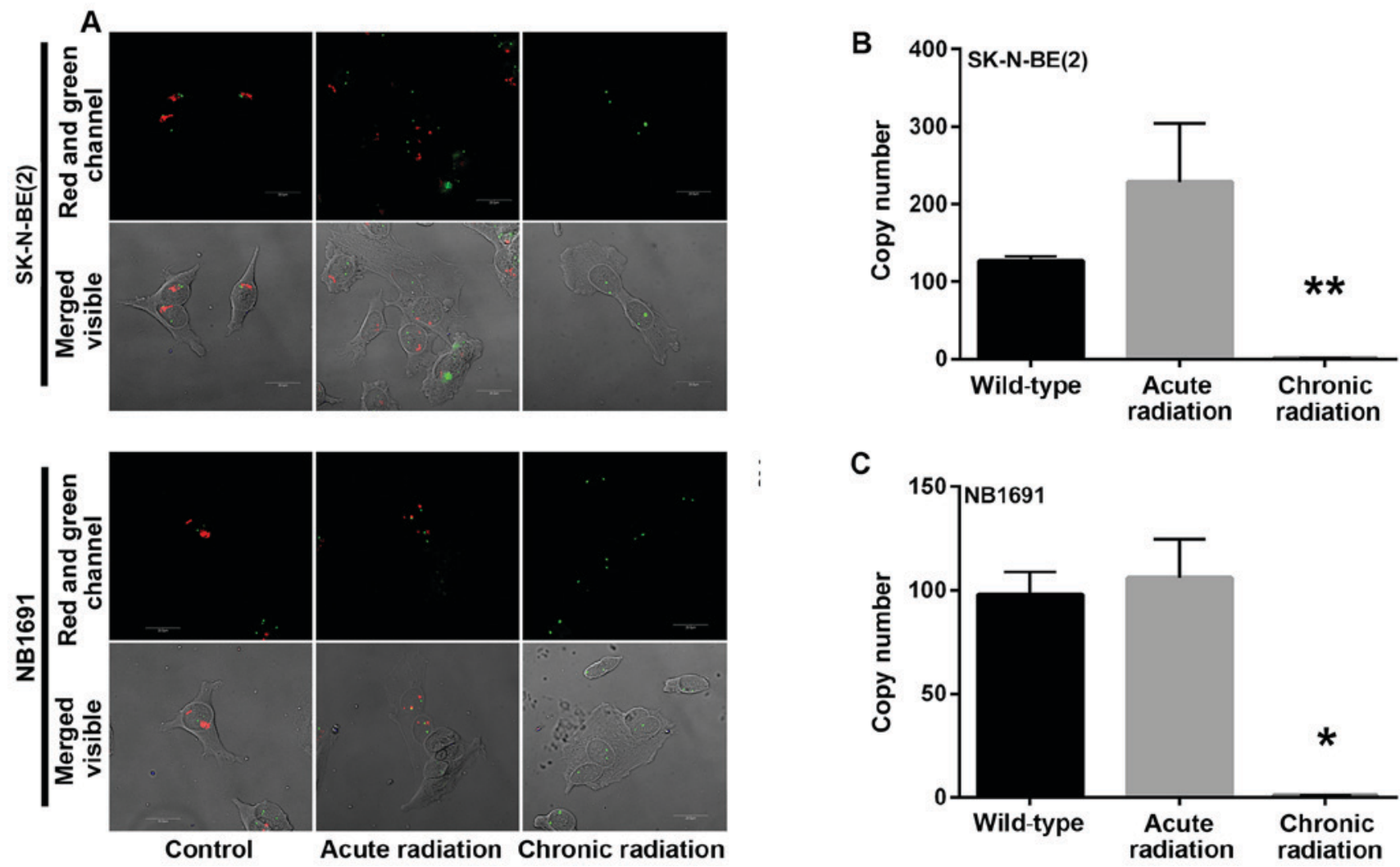

Figure 4. MYCN gene amplification analysis. (A) MYCN gene copy number was measured using FISH in neuroblastoma cells treated with acute and chronic radiation. Confocal microscopy images illustrate complete loss of nMYC gene in chronic radiation cells compared with acute radiation cells and controls. A total of two copies of the control gene LAF4 were identified clearly in the control and radiation-treated cells, which served as validation for the assay. (B and C) Quantitative polymerase chain reaction analysis using comparative $\mathrm{Ct}$ analysis demonstrates that chronic, but not acute, radiation results in a significant loss of MYCN copy number in SK-N-BE (2) and NB-1691 cells ( $\left.\mathrm{P}<0.0001 ;{ }^{* *} \mathrm{P}=0.001\right)$. Red staining indicates nMYC gene expression; green staining indicates the control gene LAF4. LAF4, lymphoid nuclear protein related to AF4; MYCN/nMYC, v-Myc avian myelocytomatosis viral oncogene neuroblastoma-derived homolog.

that cells treated with chronic but not acute radiation exhibited decreased invasive potential (Fig. 5B and C).

\section{Discussion}

In the present study, the effect of acute and chronic radiation on high-risk neuroblastoma cells was analyzed. As expected, the majority of cells treated with acute radiation did not survive and failed to proliferate efficiently, as seen by the colony formation and viability assays. In order to simulate biological and clinical radiation treatment modalities, the few cells that survived acute radiation were selected and clonally expanded. These radiation-resistant cells were additionally treated with multiple cycles of 5 Gy radiation for a total of $25 \mathrm{~Gy}$, and clonally expanded. Notably, these cells that survived chronic radiation demonstrated biological characteristics similar to those of the control untreated cells. These chronic radiation cells were able to form individual colonies and the un-radiated controls. Additionally, the results also demonstrated that these cells regained their proliferation potential, and were also proliferating at a higher rate compared with the controls. It is known that in response to radiation, several pathways are activated that target DNA repair, including cell cycle regulation, proliferation and apoptosis and other pathways (11).

Since $\mathrm{nMYC}$ has been implicated in the aggressive behavior of neuroblastoma, the present study sought to explore the expression levels of nMYC at the mRNA and protein levels. It was revealed that chronic radiation resulted in the loss of nMYC mRNA and protein expression, and that this was accompanied by a corresponding increase in the expression of cMYC. There are no concrete reports on the distinct roles of cMYC and $\mathrm{nMYC}$, and $\mathrm{nMYC}$ and $\mathrm{cMYC}$ regulate the same genes involved in the cell cycle, proliferation and death, and may partially compensate for the functions of each other (4). In murine development, nMYC has been demonstrated to successfully replace cMYC functions (12). However, in a previous study with embryonic stem cells, cMYC upregulation failed to compensate for nMYC functions (13). Additionally, it is known that in neuroblastoma there is an inverse association between nMYC and cMYC expression (14). High nMYC expression due to nMYC amplification is known to suppress cMYC expression in neuroblastoma cells (14), thereby indicating that nMYC function may be compensated for by cMYC.

To additionally explore the role of nMYC/cMYC, the present study sought to determine their subcellular localization. It was observed that $\mathrm{cMYC}$ was localized in the nucleus of SK-N-BE (2) and NB-1691 cells in response to chronic radiation, which was accompanied by a loss of nMYC expression. A previous study indicated that $\mathrm{cMYC}$ was primarily localized to the nucleus in patients with Burkitt lymphoma, whereas in patients with diffuse large B-cell lymphomas, it was observed predominantly in the cytoplasm (15). It has 

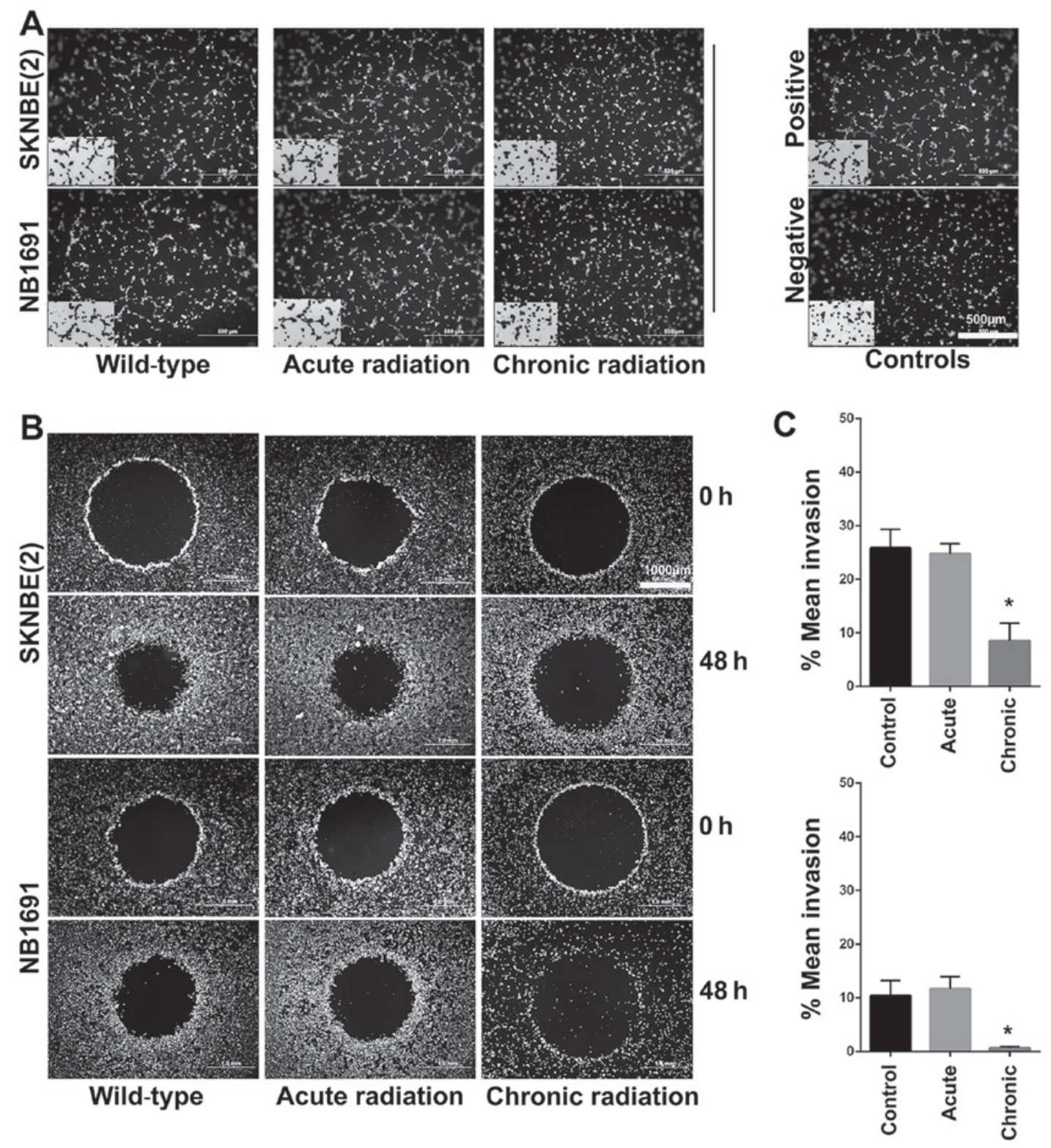

Figure 5. Role of chronic radiation on angiogenesis and invasion. (A) Angiogenesis was studied using the in vitro endothelial cell tube formation assay. Representative images illustrate decreased angiogenesis in human umbilical vein endothelial cells treated with conditioned medium from chronic radiation cells. (B) In vitro invasion was studied using the Oris Pro 96 well invasion assay kit. (C) After 48 h, invasion capability was decreased significantly in the SK-N-BE (2) and NB-1691 cells treated with chronic radiation compared with controls ( $\mathrm{P}=0.003)$.

already been demonstrated that cMYC protein possesses two nuclear localization signal regions (16). Following nuclear localization, it has been identified that $\mathrm{cMYC}$ binds to its partner Max in promyelocytic leukemia nuclear bodies and fibrillarin $(17,18)$. The present study was able to identify low levels of cMYC expression in the cytoplasm of cells treated with acute radiation. The hypothesis of the present study is that radiation upregulates the expression of cMYC protein in the cytoplasm. However, high levels of nMYC present in the nucleus prevent nuclear translocation of cMYC. During chronic radiation, loss of nMYC favors nuclear import of cMYC, which in turn activates the pathways needed for survival and proliferation.

The mechanism by which chronic radiation results in the loss of nMYC but not cMYC remains unknown. Radiation has been demonstrated to result in a significant decrease in amplified gene copy number in transformed human cell lines (17). This nMYC loss may have occurred either due to the sustained DNA damage to the cells or the loss of key DNA repair mechanisms. The underlying molecular mechanism for the specificity of this loss and gain remains to be assessed. The results of the present study are vital as there are very few studies that have documented changes in gene copy number following exposure to radiation, and this has a major impact on the future of radiation therapy.

nMYC amplification has been associated with increased angiogenesis and metastasis in children with neuroblastoma (19). In the present study, chronic radiation cells demonstrated decreased angiogenic and invasive potential. It is suggested that this may be a result of the loss of key signaling pathways regulated by nMYC. The results of the present study also support the view that certain cancer cells adapt to 
treatment pressure by switching on genes that aid dormancy. Such dormant cells with poor metastatic ability have been demonstrated to exist in neuroblastoma (20). It is hypothesized that these cells may act as precursors for metastases.

In the present study, certain important differences between acute and chronic radiation treatment of nMYC amplified neuroblastoma have been highlighted. It has been demonstrated that cells treated with chronic radiation, but not acute radiation, were able to form colonies and proliferate efficiently. Contrary to previous hypotheses, the results of the present study suggest that there is severe loss of expression of nMYC mRNA and protein in response to chronic radiation, and is compensated for by the nuclear localization of cMYC. The results of the present study also revealed that chronic radiation induces copy number changes in $\mathrm{nMYC}$ and, to the best of our knowledge, the present study provides the first experimental demonstration that indicates that $\mathrm{nMYC}$ gene copy number is severely altered following chronic radiation. Additional studies examining the exact mechanisms that result in the loss of nMYC gene copy number, and factors that drive the cells to survive, will lead the design of improved treatment strategies and the overcoming of radiation resistance.

\section{Acknowledgements}

The present study was supported by the National Cancer Institute (grant no. R01CA147792).

\section{References}

1. Kohl NE, Kanda N, Schreck RR, Bruns G, Latt SA, Gilbert F and Alt FW: Transposition and amplification of oncogene-related sequences in human neuroblastomas. Cell 35: 359-367, 1983.

2. Kohl NE, Legouy E, DePinho RA, Nisen PD, Smith RK, Gee CE and Alt FW: Human N-myc is closely related in organization and nucleotide sequence to c-myc. Nature 319: 73-77, 1986.

3. Cohn SL, Pearson AD, London WB, Monclair T, Ambros PF, Brodeur GM, Faldum A, Hero B, Iehara T, Machin D, et al: The international neuroblastoma risk group (INRG) classification system: An INRG task force report. J Clin Oncol 27: 289-297, 2009.

4. Huang M and Weiss WA: Neuroblastoma and MYCN. Cold Spring Harb Perspect Med 3: a014415, 2013.

5. Brodeur GM, Seeger RC, Schwab M, Varmus HE and Bishop JM: Amplification of N-myc in untreated human neuroblastomas correlates with advanced disease stage. Science 224: 1121-1124, 1984.

6. Gatcombe HG, Marcus RB Jr, Katzenstein HM, Tighiouart M and Esiashvili N: Excellent local control from radiation therapy for high-risk neuroblastoma. Int J Radiat Oncol Biol Phys 74: 1549-1554, 2009.

7. Morgan MA and Lawrence TS: Molecular pathways: Overcoming radiation resistance by targeting DNA damage response pathways. Clin Cancer Res 21: 2898-2904, 2015.
8. Livak KJ and Schmittgen TD: Analysis of relative gene expression data using real-time quantitative PCR and the 2(-Delta Delta C(T)) method. Methods 25: 402-408, 2001.

9. De Preter K, Speleman F, Combaret V, Lunec J, Laureys G, Eussen BH, Francotte N, Board J, Pearson AD, De Paepe A, et al: Quantification of MYCN, DDX1, and NAG gene copy number in neuroblastoma using a real-time quantitative PCR assay. Mod Pathol 15: 159-166, 2002.

10. Schweigerer L, Breit S, Wenzel A, Tsunamoto K, Ludwig R and Schwab M: Augmented MYCN expression advances the malignant phenotype of human neuroblastoma cells: Evidence for induction of autocrine growth factor activity. Cancer Res 50: 4411-4416, 1990

11. Maier P, Hartmann L, Wenz F, and Herskind C: Cellular Pathways in Response to Ionizing Radiation and Their Targetability for Tumor Radiosensitization. Int J Mol Sci 17: 102, 2016.

12. Malynn BA, de Alboran IM, O'Hagan RC, Bronson R, Davidson L, DePinho RA and Alt FW: N-myc can functionally replace c-myc in murine development, cellular growth, and differentiation. Genes Dev 14: 1390-1399, 2000.

13. Stanton BR, Perkins AS, Tessarollo L, Sassoon DA and Parada LF: Loss of N-myc function results in embryonic lethality and failure of the epithelial component of the embryo to develop. Genes Dev 6: 2235-2247, 1992

14. Breit S and Schwab M: Suppression of MYC by high expression of NMYC in human neuroblastoma cells. J Neurosci Res 24: 21-28, 1989.

15. Ruzinova MB, Caron T and Rodig SJ: Altered subcellular localization of c-myc protein identifies aggressive B-cell lymphomas harboring a c-MYC translocation. Am J Surg Pathol 34: 882-891, 2010.

16. Dang CV and Lee WM: Identification of the human c-myc protein nuclear translocation signal. Mol Cell Biol 8: 4048-4054, 1988.

17. Soldani C, Bottone MG, Biggiogera M, Alpini C, Scovassi AI, Martin T and Pellicciari C: Nuclear localization of phosphorylated c-myc protein in human tumor cells. Eur J Histochem 46: 377-380, 2002.

18. Smith KP, Byron M, O'Connell BC, Tam R, Schorl C, Guney I, Hall LL, Agrawal P, Sedivy JM and Lawrence JB: C-myc localization within the nucleus: Evidence for association with the PML nuclear body. J Cell Biochem 93: 1282-1296, 2004.

19. Meitar D, Crawford SE, Rademaker AW and Cohn SL: Tumor angiogenesis correlates with metastatic disease, N-myc amplification, and poor outcome in human neuroblastoma. J Clin Oncol 14: 405-414, 1996.

20. Edry Botzer L, Maman S, Sagi-Assif O, Meshel T, Nevo I, Bäuerle T, Yron I and Witz IP: Lung-residing metastatic and dormant neuroblastoma cells. Am J Pathol 179: 524-536, 2011. 